\title{
Caracterização de idosos participantes de atividade física em um centro de convivência de Teresina-PI
}

Recebido em: 12/08/2011

Aceito em: 15/02/2012

\author{
Nancy Nay Leite de Araújo Loiola Batista ${ }^{1}$ \\ Débora Josefina Neiva Vieira² \\ Gislene Mariana Pereira da Silva ${ }^{3}$
}

Objetivou-se a caracterização dos idosos que participam de atividade física em um centro de convivência na cidade de Teresina, assim como as relações entre as características desse perfil. Estudo descritivo de abordagem quantitativa com 234 idosos mediante aplicação de um questionário estruturado. O perfil desses idosos favorece o direcionamento de ações voltadas para a saúde, quanto aos aspectos físicos, sociais, econômicos e culturais. Portanto, um alerta para sociedade, visando à qualidade de vida, postergando o aparecimento de doenças crônico-degenerativas ou tratando-as, mantendo a capacidade funcional por meio de atividade física.

Descritores: Idoso, Atividade Motora, Enfermagem.

Characterization of elderly participants in physical activity in a social center of Teresina-PI

This study aimed to to characterize the elderly who participate in physical activity in Family Center in the city of Teresina, as well as the relationships between the characteristics of this profile. Quantitative descriptive study of 234 elderly by applying a structured questionnaire. The profile of the elderly favors the direct actions for health, regarding physical, social, economic and cultural. So a warning to society, aiming at the quality of life, delaying the onset of chronic degenerative diseases or treating them, maintaining the functional capacity by means of physical activities.

Descriptors: Aged, Motor Activity, Nursing.

\section{Caracterización de los participantes de edad avanzada en la actividad física en un centro social de Teresina-PI}

El objetivo de la investigación es la caracterización de los ancianos que participan de las actividades físicas en el centro de la família na la ciudad de Teresina así como las relaciones entre las características de este perfil. Estudio cuantitativo descriptivo de 234 personas mayores mediante la aplicación de un cuestionario estructurado. El perfil de las personas mayores favorece a las acciones directas para la salud, en cuanto a físico, social económico y cultural. Por lo tanto, una advertencia a la sociedad, con miras a la calidad de vida, retrasar la aparición de enfermedades crónico-degenerativas o el tratamiento de ellos, mantener la capacidad funcional a través de la actividad física.

Descriptores: Ancianos, La Actividad Motora, La Enfermería.

\section{INTRODUÇÃO}

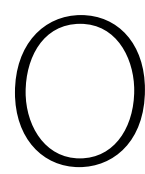
crescimento da população idosa é um fenômeno mundial. Segundo o Instituto Brasileiro de Geografia e Estatística - IBGE, constatou-se que, a cada dez brasileiros, um tinha mais de 60 anos, proporção que deverá atingir um em cada cinco brasileiros até 2050. De 1991 a 2000, o número de idosos aumentou em quase 4 milhões, atingindo $8,6 \%$ da população total(1).

A população idosa vem crescendo nos últimos anos devido às melhoras nas condições de vida e aos avanços da ciência, o que tem aumentado a expectativa de vida do ser humano. Entretanto, $\mathrm{o}$ processo de envelhecimento é acompanhado de perdas estruturais e funcionais, que favorecem o aparecimento de doenças crônicas degenerativas que podem comprometer a qualidade de vida(2).

O aumento do número de idosos na população tem se traduzido em um maior número de problemas de longa duração, seja para o individuo, seja para a sociedade. Aproximadamente 80\% das pessoas acima de 65 anos apresentam pelo menos um problema crônico de saúde. Com o avanço da idade, há um aumento progressivo da necessidade de assistência na realização de atividades da vida diária ${ }^{(3)}$.

Estudos relacionados às pessoas idosas têm demonstrado que é possível minimizar e diminuir o declínio físico-fisiológico, influenciando também nos aspectos sociais e psicológicos através de atividades físicas ${ }^{(4)}$. Hoje, tornou-se praticamente um consenso entre os profissionais da área da saúde que a atividade física é um fator determinante no sucesso do processo do envelhecimento ${ }^{(5)}$.

Existe ampla evidência demonstrando que a atividade física está associada a melhorias significativas na habilidade funcional e na condição de saúde, podendo prevenir certas doenças ou diminuir sua gravidade. Mas é importante

1 Enfermeira pela Universidade Federal do Piauí. Enfermeira da Fundação Municipal de Saúde de Teresina - Estratégia Saúde da Família. Docente do curso de enfermagem da Faculdade Santo Agostinho - FSA e Faculdade Aliança. E-mail: nancyloiola@uol.com.br.

2 Acadêmica do curso de graduação em enfermagem da Faculdade Santo Agostinho - FSA.

3 Acadêmica do curso de graduação em enfermagem da Faculdade Santo Agostinho - FSA. 
salientar que muitos desses benefícios requerem participação regular e contínua(6).

Crescentemente, observam-se pessoas com mais de 60 anos praticando exercícios em academias, clubes, associações e até sozinhos $^{(4)}$. A sociedade e a população idosa começam a ver a atividade física como forma de prevenção e reabilitação da saúde, dando maior importância a sua prática ${ }^{(7)}$.

Os programas de promoção da atividade física na comunidade, bem como a participação da população idosa nesses programas, têm crescido em popularidade nos últimos anos. Atualmente, os programas de atividade física para idosos são incentivados por diferentes órgãos oficiais que trabalham na promoção de saúde e estão sendo implantados em muitos municípios em diferentes países ${ }^{(8)}$.

Este estudo teve como objetivo caracterizar os idosos que participam de atividade física em centro de convivência na cidade de Teresina, Piauí.

\section{METODOLOGIA}

Realizou-se um estudo descritivo de abordagem quantitativa em um centro de convivência na cidade de Teresina.

A amostra não-probabilística aleatória simples, considerando um intervalo de $95 \%$ de confiança e uma margem de erro estimada em 5\%, resulta na necessidade de aplicação de 234 formulários. Os critérios de inclusão foram: ter idade igual ou superior a 60 anos, frequentar o centro de convivência e aceitar responder ao Termo de Consentimento Livre e Esclarecido.

$\mathrm{Na}$ coleta de dados, utilizou-se um formulário com quatro perguntas que respondem às características dos idosos relacionadas a identificação (idade, sexo, estado civil, escolaridade e renda familiar), tempo de atividade física no centro de convivência, frequência de atividades durante a semana e presença de algumas doenças crônicas, segundo os principais tipos.

A aplicação dos formulários ocorreu entre setembro e outubro de 2010, conforme a resolução 196/96. A pesquisa foi autorizada pela Fundação Municipal de Saúde (FMS) de TeresinaPI e pelo Comitê de Ética em Pesquisa (CEP) da Faculdade Santo Agostinho (FSA).

A análise dos dados foi possível após a construção de gráficos em forma de coluna, a partir dos dados tabulados, com o auxílio do software Excel versão 2007.

\section{RESULTADOS E DISCUSSÃO}

As características dos idosos frequentadores de um centro de convivência na cidade de Teresina-PI revelam o predomínio de participantes na faixa etária de 66-70 anos, sexo feminino, viúvos, renda mensal de até um salário mínimo, escolaridade primária (fundamental incompleto), participação das atividades há mais de cinco anos, com frequência de três vezes na semana e a presença de hipertensão arterial.

Os resultados encontrados no gráfico 1 apontam a proporção de idosos participantes de atividade física em um centro de convivência de Teresina-PI segundo a faixa etária. Observa-se que a maioria $(39,8 \%)$ dos participantes do estudo é de idosos com idade entre 66 e 70 anos, o que, segundo Papália(9), define esse grupo como "velhos jovens", geralmente ativos, joviais e vigorosos.

Nessa faixa etária, encontramos idosos fora do mercado de trabalho, portanto com mais tempo livre para as atividades dentro do centro de convivência. Esses, sobretudo, apresentam maior consciência dos limites da idade. Segundo Acosta(10), os antigos estereótipos do envelhecimento não são mais adotados pelos idosos. Eles não aceitam mais "ficar em casa cuidando dos netos" ou fazendo crochê; buscam novas atividades que tragam maior bem-estar.

De acordo com o sexo, os resultados do gráfico 2 identificam a grande participação do gênero feminino, correspondendo a $87,6 \%$, confirmando a feminização da velhice.

A diferença de gênero na participação de atividades do centro de convivência é justificada pela maior consciência das mulheres sobre a necessidade de realizar atividades físicas para uma melhor qualidade de vida. De outro modo, o homem, que passou praticamente a vida toda fora de casa trabalhando, com

Gráfico 1: Proporção de idosos participantes de atividade física em um centro de convivência, segundo a faixa etária. Teresina$\mathrm{Pl}$, set/out 2010

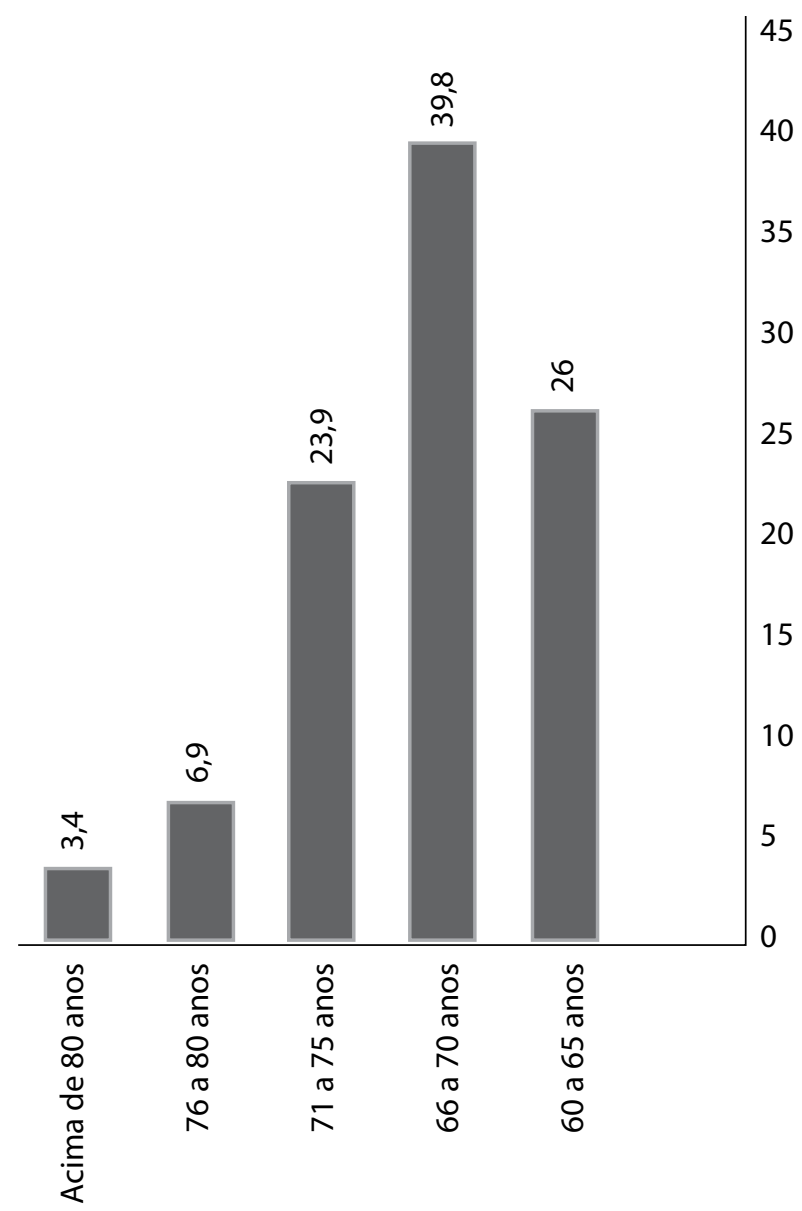


Gráfico 2: Proporção de idosos participantes de atividade física em um centro de convivência, segundo o sexo. Teresina-PI, set/ out 2010

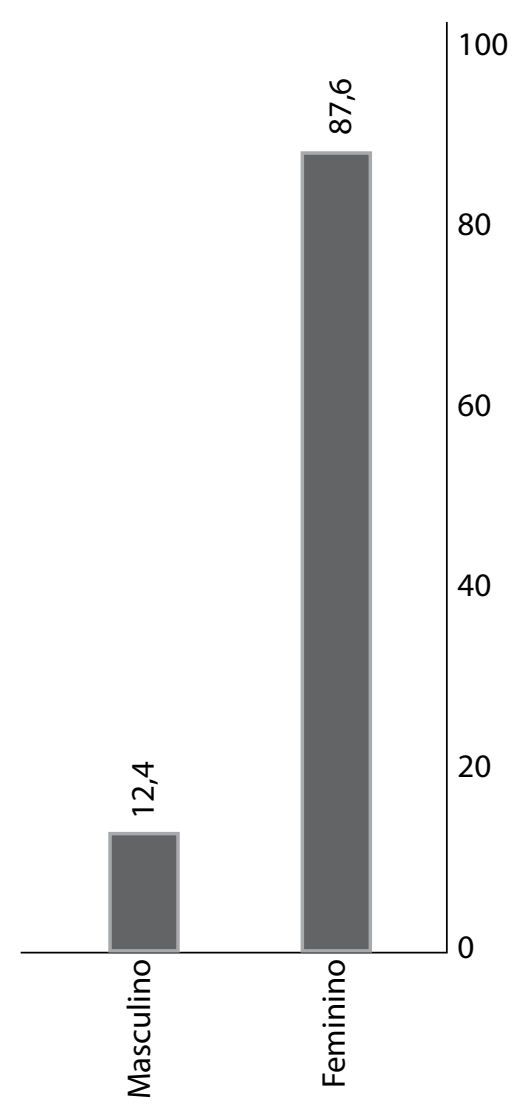

a chegada da aposentadoria, prefere passar mais tempo em casa, saindo para ver os amigos ou se distrair(11).

$\mathrm{Na}$ velhice, muitos homens são levados a procurar ajuda médica diante de quadros irreversíveis de adoecimento, por não terem lançado mão de ações de prevenção ou de tratamento precoce das enfermidades ${ }^{(12)}$. Portanto, ações destinadas aos grupos de convivência de idosos devem considerar o universo feminino na terceira idade e peculiaridades, ao mesmo tempo que considerem alternativas que atraiam os homens, favorecendo a integração social, informação, lazer e qualidade de vida.

Pode ser observado no gráfico 3 que a maioria dos idosos é constituída de viúvos $(31,2 \%)$ e que, nesse grupo, a viuvez se constata como exclusividade das mulheres, pois todos os homens abordados na pesquisa eram casados. Em quase todos os países, o número de viúvas é maior do que o de viúvos, principalmente em países desenvolvidos(13).

As mulheres têm tendência a viver sozinhas ou continuar viúvas. Já os homens se casam novamente. Nesse contexto, muitas relatam solidão, mas outras consideram a viuvez como possibilidade de ter liberdade e autonomia.

A busca pelo centro de convivência pode ser justificada pelo preenchimento do vazio deixado pelo companheiro, pois nesses
Gráfico 3: Proporção de idosos que declararam seu estado civil atual. Teresina-PI, set/out 2010

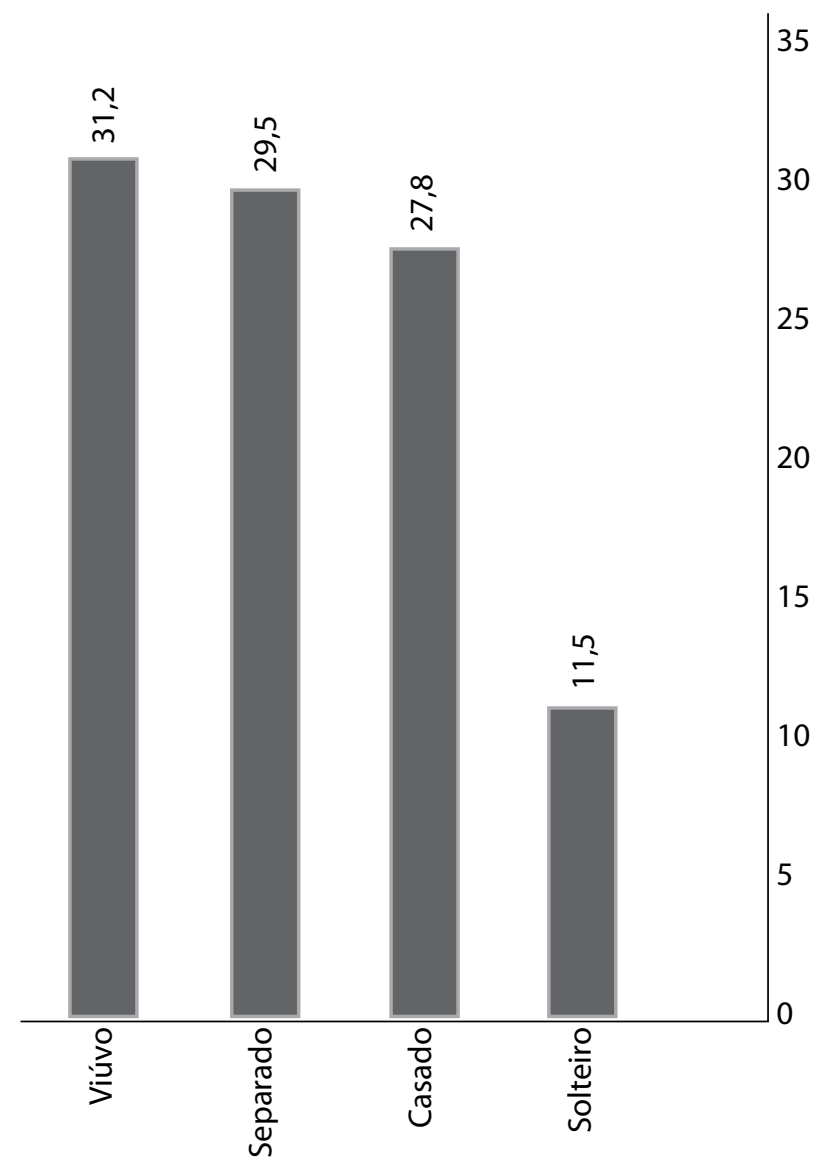

locais convivem com pessoas da mesma faixa etária e problemas parecidos, podendo trocar experiências e renovar amizades.

No gráfico 4, observa-se a proporção dos idosos quanto à renda familiar em salários mínimos. O resultado corresponde a um pouco mais da metade dos idosos $(56,8 \%)$ que recebem até um salário mínimo por mês, por aposentadoria ou pensão.

As políticas de envelhecimento ativo precisam cruzarse com projetos mais amplos para reduzir a pobreza em todas as idades. Os pobres de todas as idades apresentam um risco maior de doenças e deficiências, e os idosos estão particularmente vulneráveis. Muitos idosos, especialmente as mulheres, vivem sozinhos ou em áreas rurais sem renda certa ou suficiente. Esses fatores afetam seriamente o acesso a alimentos nutritivos, a moradias adequadas e a cuidados de saúde.

Na verdade, alguns estudos demonstraram que idosos com baixa renda têm uma chance de cerca de $30 \%$ de apresentar altos níveis funcionais se comparados àqueles que possuem uma renda alta(14). As idosas de baixa renda executam sozinhas atividades domésticas como cozinhar, varrer, lavar roupas, sobretudo se moram sós ou com os filhos, e ainda ajudam a cuidar das crianças. Esse fato ocorre por não terem 
condições de contratar alguém para executar tais atividades, e em virtude dessas condições continuam por mais tempo ativas, contribuindo de forma positiva na sociedade em que estão inseridas.

De acordo com o nível de escolaridade apresentado pelos idosos do local do estudo, quase a metade da população idosa $(40,2 \%)$ cursou até as primeiras séries do ensino fundamental, não completando esse nível escolar. A baixa escolaridade encontrada está de acordo com uma série de estudos de base populacional, mas pode associar-se também ao tipo de atividade oferecida nos grupos de convivência de idosos, que não requer escolaridade mínima para participação e entendimento.

Assim, no desenvolvimento de atividades para os grupos de convivência de idosos, além de considerar a adequação para a baixa escolaridade dos idosos, deve-se pensar na inserção de programas que possibilitem novas formas de acesso à educação formal e informal, com metodologia adequada às necessidades desse público.

Quanto ao tempo de prática de atividade física no centro de convivência, cerca de 40,2\% dos idosos abordados frequentam o local há mais de cinco anos. Com base nesse dado, sugerese que os mesmos estão encontrando o que buscam nesses locais e confirmam a possibilidade de utilização dos grupos de

Gráfico 4: Proporção dos idosos quanto à renda familiar em salários mínimos. Teresina-PI, set/out 2010

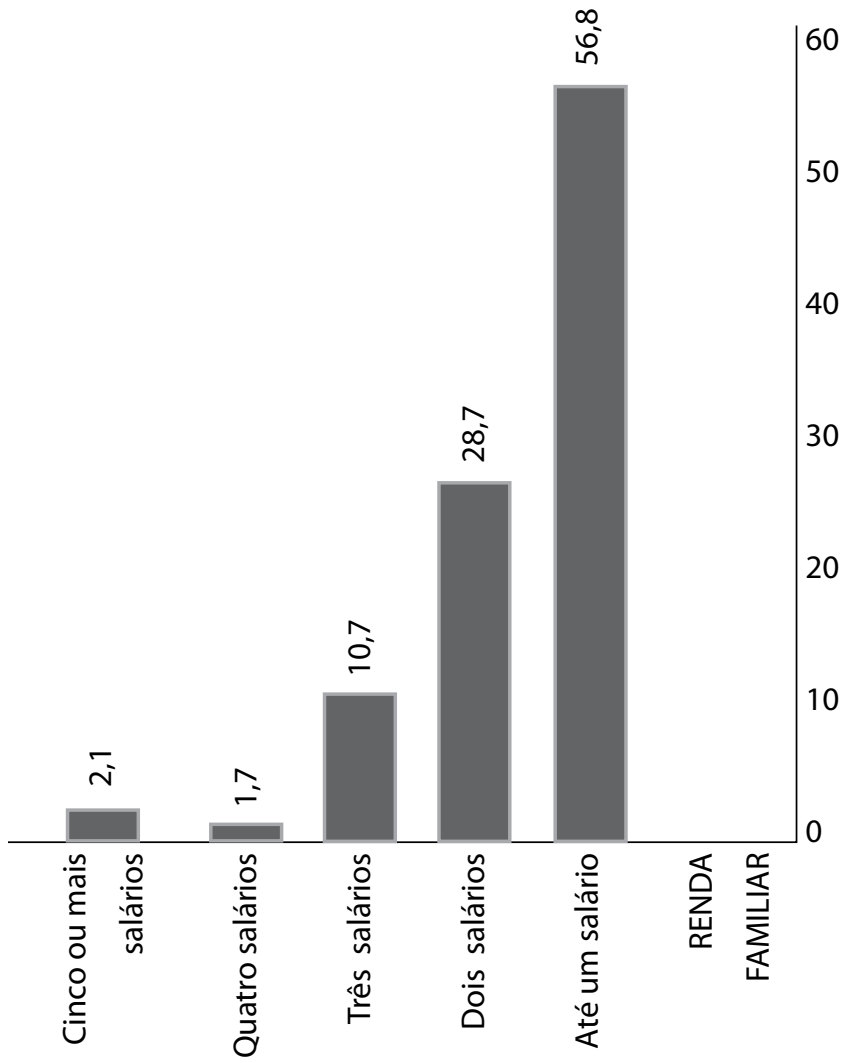

Gráfico 5: Doenças crônicas que mais acometem os idosos do CCTI, segundo os principais tipos. Teresina-PI, set/out 2010

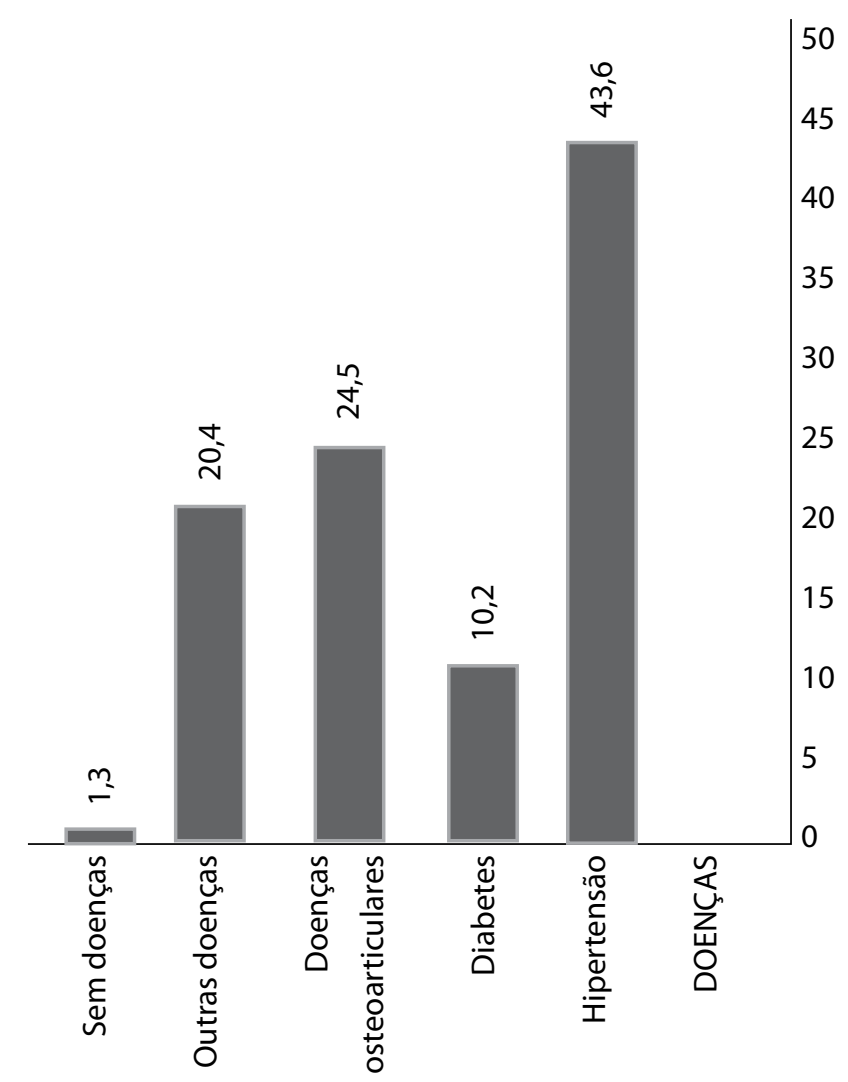

convivência como veículos para aumentar a qualidade de vida e a oferta de serviços aos idosos ${ }^{(15)}$.

Segundo Gaspari e Schwartz ${ }^{(16)}$, os indivíduos idosos procuram atividades de lazer, ampliação do rol de amizades, conhecimento de lugares novos, convivência e troca de experiências de vida com outras pessoas. Por essas razões, os idosos frequentam o CCTI várias vezes durante a semana. A frequência maior nas atividades oferecidas pelo centro de convivência é de pelo menos três vezes na semana, correspondendo a 45,3\% dos idosos.

Os principais problemas de saúde relatados pela maioria dos idosos é a hipertensão arterial (43,6\%), seguida das doenças osteoarticulares - artrite, artrose, osteoporose (24,5\%), representado no gráfico 5 .

A hipertensão arterial (HA) é o distúrbio cardiovascular de maior prevalência no Brasil. Mais de $50 \%$ de toda população com mais de 60 anos tem hipertensão arterial. É a principal etiologia nas doenças coronarianas, cérebro-vasculares e reno-vasculares ${ }^{(13)}$.

Segundo Barroso ${ }^{(17)}$, a hipertensão arterial é uma das doenças de maior prevalência na população adulta e principalmente nos idosos. Está associada ao aumento na morbidade-mortalidade por todas as causas cardiovascular e os programas de exercício estão associados à prevenção do desenvolvimento de hipertensão e também à redução da PA. 
Esses resultados sugerem que os grupos de convivência de idosos podem ser considerados importantes veículos para informações sobre a saúde do idoso, visando a informar sobre a prevenção e o controle dos principais problemas de saúde do envelhecimento.

\section{CONCLUSÃO}

Os resultados indicam que o grupo de convivência de idosos em estudo é frequentado principalmente por mulheres, viúvas, de baixa renda e escolaridade. Os idosos que participam do centro de convivência correspondem aos chamados "velhos jovens", que são geralmente mais ativos, joviais e vigorosos. Apesar de a maioria ser de velhos mais jovens, esses não estão isentos de apresentar algum problema de saúde. O problema de saúde que mais acomete o grupo é a hipertensão, seguida de doenças osteoarticulares (artrite, artrose, osteoporose).

Esse perfil social e epidemiológico indica que os grupos de convivência de idosos podem ser importantes veículos para que as ações de saúde atinjam um número significativo de idosos. Essa caracterização deve ser considerada na programação e implementação das medidas de saúde e geração de renda para idosos da comunidade e para a adequação desses locais para que possam não só atender os participantes, mas também outros idosos que poderão se beneficiar de suas atividades.

O levantamento do perfil daqueles que participam de grupos de convivência de idosos poderia favorecer o direcionamento de ações tanto no plano da saúde quanto nos aspectos físicos, sociais, emocionais, culturais e econômicos, além de delinear as necessidades de adequação desses locais e de suas atividades para o público frequentador.

Adicionalmente, o conhecimento das características dos idosos que frequentam esses locais poderia contribuir para ações visando à participação de mais idosos. Do ponto de vista da saúde pública, investir na qualidade de vida de idosos da comunidade, para mantê-los em uma situação de envelhecimento ativo, deveria ser considerado fundamental para reduzir a necessidade de investimentos em prevenções secundária e terciária, em tratamentos, reabilitação e institucionalização.

\section{Referências}

1. Instituto Brasileiro de Geografia e Estatística. Perfil dos idosos responsáveis pelos domicilios no Brasil [Internet, citado em 2010 mar 25]. Disponivel em: http:// www.ibge.gov.br.

2. Mendonça T. Risco cardiovascular, aptidão física e prática de atividade física de idosos de um parque de São Paulo [Internet, citado em 2010 mar 10]. Disponível em: http://portalrevistas.ucb.br/index.php/RBCM/article/.

3. Pedrazzi EC. Morbidade referida a capacidade funcional de idosos. Ciênc Cuid Saúde. 2007;6(4):407-13.

4. Kuwano VG, Silveira AM. A influência da atividade física sistematizada na autopercepção do idoso em relação às atividades da vida diária [Internet, citado em 2010 mar 22]. Disponível em: http://periodicos.uem.br/ojs/index.php/ RevEducFis/article/.

5. Matzudo SM. Atividade física e envelhecimento: aspectos epidemiológicos. Rev Bras Med Esporte. 2001;7(1).

6. Organización Mundial de la Salud. The Heindelberg Guidelines for promoting physical activity among older persons [Internet, citado em 2010 mai 02]. Disponivel em: www.who.dk/document/e75205.pdf.

7. Freitas EV. Tratado de geriatria e gerontologia. $2^{\mathrm{a}}$ ed. Rio de Janeiro: Guanabara Koogan; 2006.

8. Benedetti TRB, Gonçalves LHT, Mota JAPS. Uma proposta de política pública de atividade física para idosos. Texto Contexto Enferm. 2007;16(3):387-98.

9. Papalia DE, Olds SW, Feldman RD. Desenvolvimento humano. 8 a ed. Porto
Alegre: Artmed; 2006.

10. Acosta MAF. Getting old in the city: a study on groups of physical activities for elderly people in Santa Maria/RS. FIEP Bulletin. 2007;77:625-8.

11. Coutinho RX, Santos $C M$, Acosta MAF. Perfil de idosos praticantes de atividades fisicas: saúde da vida diária [Internet, citado em 2010 nov 25]. Disponível em: http://www.efdeportes.com/efd133/idosos-praticantes-deatividades-fisica.

12. Ministério da Saúde (BR). Política Nacional de Atenção Integral à Saúde do Homem: princípios e diretrizes. Brasilia: Ministério da Saúde; 2009.

13. Saldanha AL, Caldas CP. Saúde do idoso: a arte de cuidar. $2^{\mathrm{a}}$ ed. Rio de Janeiro: Interciência; 2004.

14. Organización Mundial de la Salud. World Health Organization. Envelhecimento ativo: uma política de saúde. Brasilia: Organização Pan-Americana da Saúde; 2005.

15. Borges PLC. Perfil dos idosos frequentadores de grupos de convivência em Belo Horizonte, Minas Gerais, Brasil [Internet, citado em 2010 nov 28]. Disponível em: http://www.scielosp.org/pdf/csp/v24n12/08.pdf.

16. De Gaspari JC, Schwartz GM. O idoso e a ressignificação emocional do lazer. Psicol Teor Pesqui. 2005;21(1):69-76.

17. Barroso WKS, Jardim PCBV, Vitorino PV, Bittencourt A, Miquetichuc F. Influência da atividade física de idosos hipertensos sob tratamento não-farmacológico [Internet, citado em 2010 nov 28]. Disponível em: http://www.scielo.br/pdf/ramb/ v54n4/17.pdf. 